\title{
Zebrafish early methylomes
}

Totipotency - the ability to differentiate into any cell type - is the platform from which the zygote is able to make all developmental decisions. How totipotency is set up is understood to some extent in mammals, in which it involves substantial reprogramming of the male and female gamete epigenetic marks, but it is not known whether the mechanisms extend across vertebrates. Two recent papers have now characterized the early DNA methylation changes in zebrafish embryos and show, surprisingly, that the DNA methylation pattern in zebrafish sperm resembles that of the zygote.

In mice, bulk demethylation after fertilization occurs at the one-cell stage and is followed by several distinct stages of methylation changes on both maternal and paternal genomes. Thus, in mice, the pathway to totipotency involves reprogramming of the male and female genomes.

To assess whether the mammalian mechanism is conserved in zebrafish, Potok et al. and Jiang et al. characterized the genome-wide pattern of DNA methylation in zebrafish gametes and at various stages in early development using whole-genome shotgun bisulphite MethylC-seq. They found that the sperm genomewide methylation profile was remarkably similar to the profile of the zygote at the stage of zygotic genome activation, whereas the oocyte profile was distinct from both that of the sperm and of the zygote at this stage.

The authors found that in the oocyte after fertilization, passive demethylation occurs at many key early developmental genes, and active methylation occurs at many other genes, such as those that are involved in mid to late development. Thus, the maternal genome is altered to

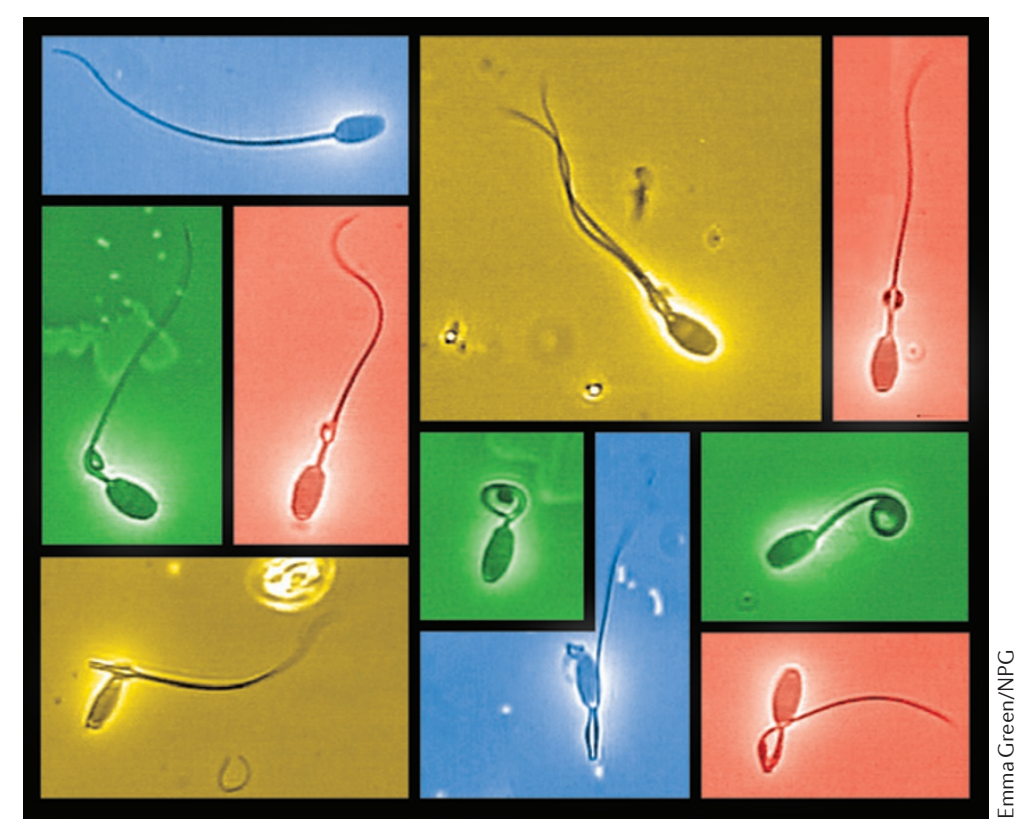

resemble that of the sperm, and there are sites at which DNA methylation changes seem to be crucial for developmental regulation; Potok et al. found these sites to be altered later on in differentiated tissue. However, Potok et al. also found evidence to support the idea that a sperm 'template' is not required for resetting the maternal genome methylation patterns, although the sperm nucleus is required.

In early development in mammals, DNA demethylation passes through a hydroxycytosine $(5 \mathrm{hmC})$ intermediate. However, neither of the groups was able to find evidence for this during DNA demethylation in zebrafish.

To assess the importance of the methylation changes in gene regulation, both groups carried out RNA sequencing (RNA-seq) in the early embryos. Hypomethylated promoters were associated with expressed genes, and hypermethylated regions were associated with silenced genes. Potok et al. also showed that inhibition of the DNA methyltransferases at the MBT stage caused alterations in the DNA methylation, resulting in precocious transcription. Thus, the methylation changes were shown to be crucial in gene regulation.

These data have shown a major difference in the way that zebrafish and mammalian embryos are programmed to totipotency. Furthermore, the potential inheritance of the epigenetic marks of the sperm is likely to spur future research, both into the mechanisms by which this occurs and into whether this is conserved in other species.

Hannah Stower

ORIGINAL RESEARCH PAPERS Potok M. E. et al. Reprogramming the maternal zebrafish genome after fertilization to match the paternal methylation pattern. Cell 153, 759-772 (2013)| Jiang, L. et al. Sperm, but not oocyte, DNA methylome is inherited by zebrafish early embryos. Cell 153, 773-784 (2013) 